\title{
IMPORTANCE OF MOUTH GUARDS IN SPORTS: A REVIEW
}

Priyadarshani G Pawar ${ }^{1}$, Mukesh M. Suryawanshi², Ashishkumar K. Patili3, Pravin S Purnale ${ }^{4}$, Fareedi Mukram Ali ${ }^{5}$

\section{HOW TO CITE THIS ARTICLE:}

Priyadarshani G Pawar, Mukesh M Suryawanshi, Ashishkumar K Patil, Pravin S Purnale, Fareedi Mukram Ali. "Importance of mouth guards in sports: a review". Journal of Evolution of Medical and Dental Sciences 2013; Vol. 2,

Issue 46, November 18; Page: 8903-8908.

ABSTRACT: Teens and adults experience thousands of injuries on the playing field, while biking and during other activities. Injuries to the face in nearly every sport can harm teeth, lips, cheeks and tongue. A properly fitted mouth protector is important to protect teeth and smile. This article gives a brief review on the mouth guards to be used to protect smile.

KEY WORDS: mouth guard, mouth protector

INTRODUCTION: Sports have the potential to seriously harm the head, face or mouth as a result of head-to- head contact, hazardous falls, tooth clenching or blow to the mouth. Knowing how to prevent injuries is important if you participate in organized sports or other recreational activities. When it comes to protecting your mouth, a mouth guard is an essential piece of athletic gear that should be part of an athlete's standard equipment from an early age. In fact, an athlete is 60 times more likely to suffer harm to the teeth when not wearing a mouth guard. Mouth guards help buffer an impact or blow that otherwise could cause broken teeth, jaw injuries or cuts to the lip, tongue or face. Mouth guards also may reduce the rate and severity of concussions. Sport, leisure and recreation activities are the most common cause of dental injuries. Dental injuries can be painful, disfiguring and expensive to treat. Dental injuries may result in time off work or school to recover, and lengthy (and expensive) dental treatment. A mouth guard, custom-fitted by your dentist and worn every time you play or train, will protect against dental injury.

HISTORY: The exact origins of the mouth guard are unclear. Most evidence indicates that the concept of a mouth guard was initiated in the sport of boxing. Originally, boxers used to wear mouth guards out of cotton, tape, sponge, or small pieces of wood. They bite the material between their teeth.(1) These devices proved impractical, a British dentist, began to fabricate mouthpieces for boxers in 1892. Krause placed strips of a natural rubber resin, gutta-percha, over the maxillary incisors of boxers. (2) Philip Krause was an amateur boxer used his own device before 1921.(3) In the early 1900s, Jacob Marks created a custom fitted mouth guard in London.(4) In 1927 boxing match between Jack Sharkey and Mike McTigue. McTigue was winning for most of the fight, but a chipped tooth cut his lip, and he was forced to forfeit the match. From that point on, mouth guards were acceptable. $(4,5)$ In 1947, a Los Angeles dentist, made a breakthrough by using transparent acrylic resin to form an "acrylic splint". In the 1948 issue of the Journal of the American Dental Association, the procedure for making and fitting the acrylic mouth guard was described in detail by Dr. Lilyquist.(6) He was awarded nationwide as the father of the modern mouth guard for athletes. (6,7) In the 1940 s and 1950s, dental injuries were responsible for $24-50 \%$ of all injuries in American football. In 1952, Life magazine did a report on Notre Dame football players without incisors.(8) In the 1950s, the American Dental Association (ADA) began conducting research on mouth guards and 
soon promoted to the public.(9) In 1960, the ADA recommended the use of latex mouth guards in all contact sports. The National Collegiate Athletic Association (NCAA) followed suit in 1973 and made mouth guards mandatory in college football. Since the introduction of the mouth guard, the number of dental injuries has decreased dramatically.(10) Mouth guards have become a standard in many sports.

\section{SPORTS WHICH NEED MOUTHGUARD:}

\begin{tabular}{|c|c|c|}
\hline \multicolumn{2}{|c|}{ The ADA recommends mouth guards be used in } \\
\hline Acrobatics & Ice Skating & Water polo \\
\hline Basketball & Inline Skating & Weight Lifting \\
\hline Bicycling & Lacrosse & Wrestling \\
\hline Boxing & Martial Arts & Volleyball \\
\hline Equestrian Events & Racquetball & Squash \\
\hline Extreme Sports & Rugby & Surfing \\
\hline Field Hockey & Shot putting & Soccer \\
\hline Football & Skateboarding & Softball \\
\hline Gymnastics & Skiing & Skydiving \\
\hline Handball & & \multicolumn{2}{|c}{} \\
\cline { 1 - 2 } & &
\end{tabular}

Common Dental Injuries And Risk factors: Common Dental Injuries Incurred During Sport Or Leisure Activities Include Cut lips, Cut gums, Cut cheeks, Cuts to the tongue or face, Chipped teeth, Broken teeth, Knocked out teeth, Broken jaw, Temporomandibular joint fractures. Some people are at higher risk of dental injury. Risk factors include: Protrusive front teeth, Inadequate lip coverage over the front teeth, Current orthodontic treatment, such as wearing braces .Participation in sports or leisure activities that carry a high risk of collision or falls. In 1995 Dr. Raymond Flanders st reported on the high incidence of injuries in sports other than football, in both male and female sporting activities. In football where mouth guards are worn, . $07 \%$ of the injuries were orofacial. In basketball where mouth guards are not routinely worn, $34 \%$ of the injuries were orofacial. Various degrees of injury, from simple contusions and lacerations to avulsions and fractured jaws are being reported. Dental injuries can be painful, disfiguring and expensive to treat. Dental injuries may result in time off work or school to recover, and lengthy (and expensive) dental treatment. Without using mouth guards, the athlete increases their chance of injury, especially concussion, from a blow to the chin. Some of these injuries, such as concussion, can cause lifelong effects.

Mouth guard features: Wearing an appropriately designed and made mouth guard while participating in sport will protect against dental injuries. Players of all ages involved in sports and activities where they are at risk of an injury to the face should protect their teeth with a properly 
fitted mouth guard. A protective mouth guard should be comfortable yet tight-fitting, allows normal breathing, speech and swallowing. It does not cause gagging or irritation. It should be odourless and tasteless and thick enough $(4 \mathrm{~mm})$ to provide protection against impact. The mouth guards were made of the following materials: (1) Poly (vinyl acetate-ethylene) copolymer clear thermoplastic (2) Polyurethane (3) Laminated thermoplastic.

Types of Mouth guards: The Three Basic Types Of Mouth guard Include:

- The Ready-Made Or Stock Mouth guard

- The Mouth- Formed "Boil-And- Bite" Mouth guard

- $\quad$ The Custom-Fitted Mouth guard --- A) The Vacuum Mouth guard

B) Pressure Laminated Mouth guard.

Stock - a ready-to-wear mouth guard that comes pre-formed. The stock mouth guard, available at most sporting good stores, come in limited sizes and are the least expensive and least protective. The stock mouth guard is the least acceptable. This type of mouth guard is often altered and cut by the athlete in an attempt to make it more comfortable, further reducing the protective properties of the mouth guard. these are the least expensive mouth guard, they also offer the least amount of protection. They tend to be uncomfortable and fit poorly.(fig.1)

Boil-and-bite - once the lining is softened in boiling water, the person bites on the mouth guard to help it take the shape of their mouth. These mouth guards may not conform to the person's bite and can be uncomfortable to wear. Athletes also cut and alter these bulky and ill fitting boil and bite mouth guards due to their poor fit, poor retention, and gagging effects ${ }^{11}$.(fig.2)

Custom-fitted - It considered to provide the best protection for the teeth, lips and jaw. Customfitted mouth guards are made by a dentist or a dental technician to fit the individual's mouth. They provide the best protection due to their close fit, comfort and cushioning (shock absorption) effect. They are the most expensive option. However, the cost of an injury to the teeth or jaw will be a lot more expensive. Dentists recommend custom-fitted mouth guards. There are two categories of custom mouth guards, the Vacuum Mouth guard and the Pressure Laminated Mouth guard.

It is made from a stone cast of the mouth, usually of the maxillary (upper) arch, using an impression by dentist. A thermoplastic mouth guard material is adapted over the cast with a special vacuum machine which will chemically fuse under high heat and pressure with machines such as the Drufomat, the Erkopress 2004, or the Biostar. (fig. 3, 4) The most common material for this use is a poly-EVA (ethylene vinyl acetate) copolymer. (fig. 5) The mouth guard is then trimmed and polished to allow for proper tooth and gum adaptation. (fig. 6) All posterior teeth should be covered and muscle attachments unimpinged. (fig. 7, 8) It should be noted that these mouth guards are still superior to the store bought stock and boil and bite mouth guards because they have a much better fit, made from a mold of your mouth, and are designed by dentist.

How To Care For Mouth guard - It include:

- Rinse the mouth guard in soap and warm water after each use. Allow it to air-dry.

- Disinfect the mouth guard from time to time with a mouthwash. 
- Keep the mouth guard in a well-ventilated plastic storage box when not in use. The box should have several holes in it.

- Do not leave the mouth guard in direct sunlight, in a closed car or in the car's glove box. Heat can damage it.

- Ensure your mouth guard is in good condition before each use.

- Ask your dentist to inspect your mouth guard at every dental check-up.

- Replace the mouth guard if it is damaged.

- Replace a child's mouth guard every 12 to 18 months, even if it appears to be in good condition. Growth and new teeth can alter the fit.

- Replace an adult's mouth guard after dental treatment or tooth loss. Otherwise it should last for several years.

- Wear the mouth guard at all times, including games and training sessions.

- Players undergoing dental treatment can have a custom-fitted mouth guard made by their dentist to fit comfortably and accurately over their braces.

DISCUSSION: As sports dentists and health professionals, we highly recommend the custom made mouth guard, especially those of the laboratory lamination type for the very best in oral/facial protection as well as concussion deterrence. This section has presented a discussion of the various issues relating to injury prevention and mouth guards. By acknowledging these significant differences in mouth guards, the public will be better informed and educated to seek their dental sports protection from dental health professionals.

CONCLUSION: Sport, leisure and recreation activities are the most common cause of dental injuries. A mouth guard, custom-fitted by your dentist, is considered to provide the best protection for the teeth, lips and jaw. The cost of an injury to the teeth or jaw far exceeds the cost of a custom-fitted mouth guard.

\section{REFERENCES:}

1. Knapik J. J., Marshall S. W., Lee R. B., Darakjy S. S., Jones S. B., Mitchener T. A., \& Jones, B. H. Mouth guards in Sport Activities. J Sports Medicine 2007;37(2), p.120.

2. Reed, R. V. Origin and early history of the dental mouthpiece. British Dental Journal $1994 ; 176$ : p. 479.

3. Knapik et al., 2007: p. 120.

4. Pontsa, Peter T. (2008). Mouth Guards Prevent Dental Trauma in Sports. The Dent-Liner 2008;12 (3).

5. Knapik et al., 2007 :p. 121.

6. "Acrylic Splints for Athletes: Transparent Slip Casings for the Teeth as a Protection From Blows." Journal of the American Dental Association 36.1 (1948) 109-110.

7. "Protecting Athletes' Teeth." Pittsburgh Post-Gazette 1948 :21.162-18.

8. Using Mouth guards to Reduce the Incidence and Severity of Sports-related Oral Injuries." Journal of the American Dental Association 137.12 (2006): 1712-1720. 


\section{REVIEW ARTICLE}

9. Zadik Y, Jeffet $U$, Levin L . "Prevention of dental trauma in a high-risk military population: the discrepancy between knowledge and willingness to comply". Mil Med 2010; 175 (12): 10001003.

10. Zadik Y, Levin L. "Orofacial injuries and mouth guard use in elite commando fighters". Mil Med 2008;173 (12): 1185-1187.

11. Zadik Y, Levin L. "Does a free-of-charge distribution of boil-and-bite mouth guards to young adult amateur sportsmen affect oral and facial trauma?". Dent Traumatol 2009; 25 (1): 69-72.

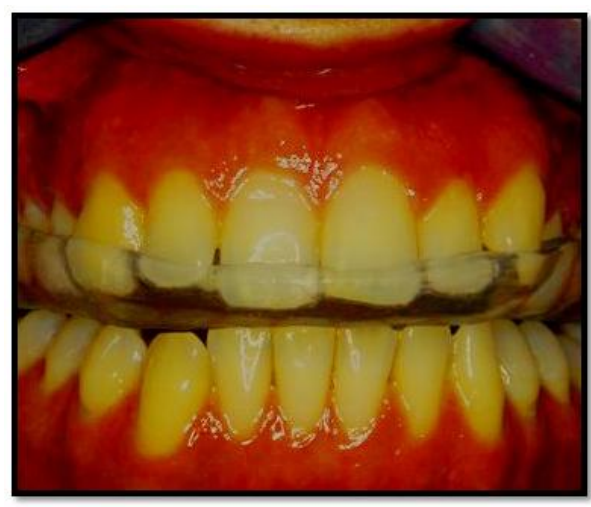

Fig. 1: Stock mouth guard
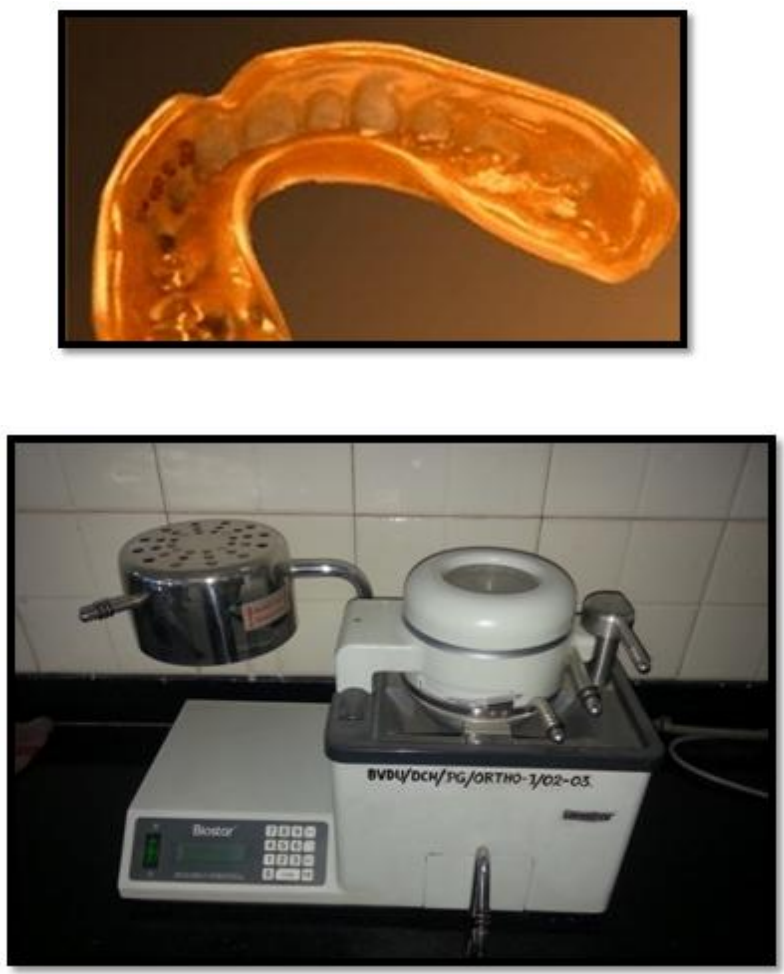

Fig. 3: Biostar vaccum machine

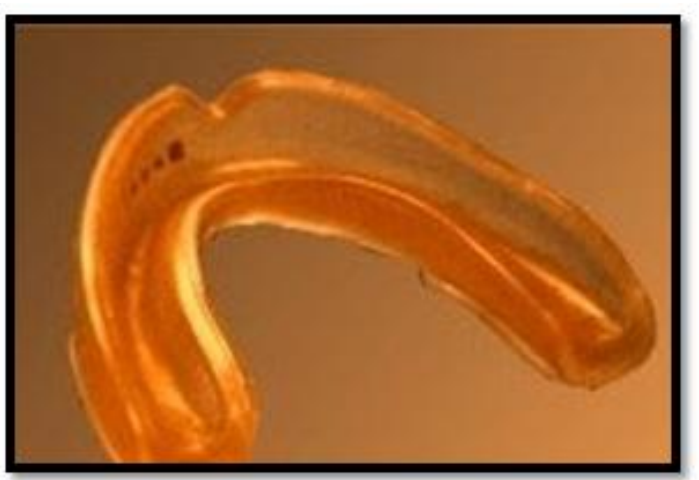

Fig. 2 (A) Boil and bite mouth guard- before fitting

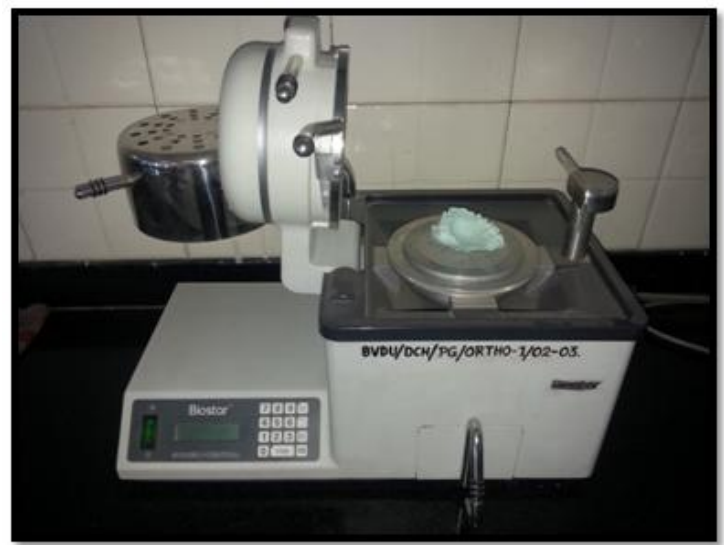

Fig.4: Fabricate a Biostar vinyl base on the cast 


\section{REVIEW ARTICLE}

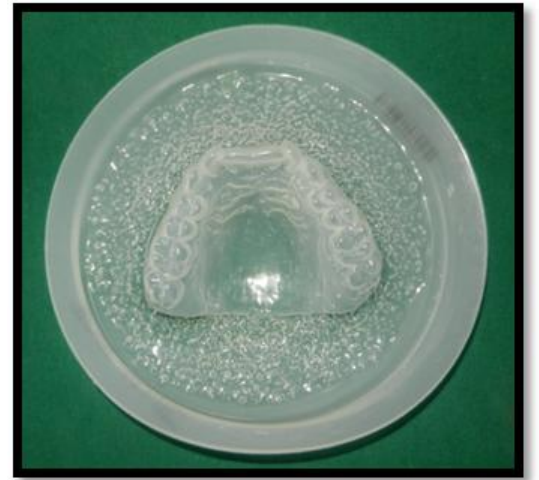

Fig. 5: Bio-star vinyl sheet after fabrication

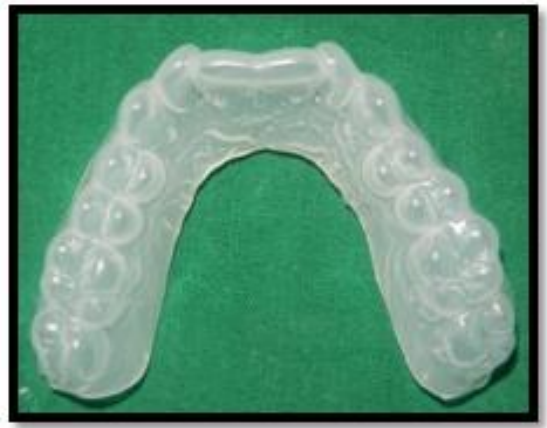

Fig.7 Custom fitted sports mouth guard prepared

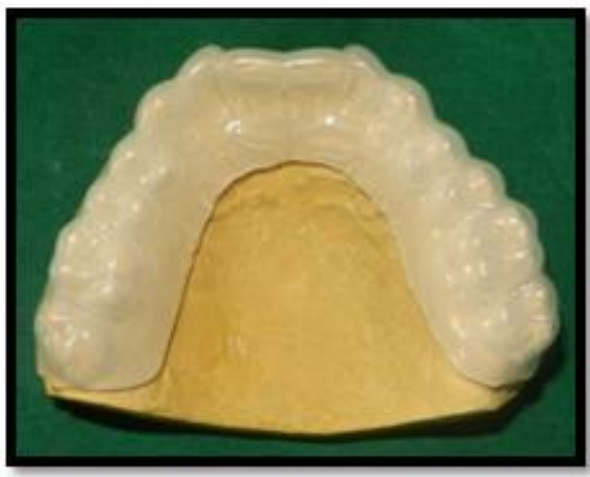

Fig.6: Try-in on cast after removal of excess

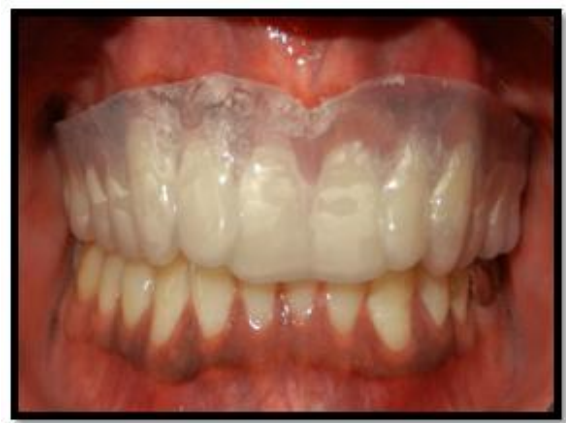

Fig. 8: Intraoral view

\section{AUTHORS:}

1. Priyadarshani G. Pawar

2. Mukesh M. Suryawanshi

3. Ashishkumar K. Patil

4. Pravin S Purnale

5. Fareedi Mukram Ali

\section{PARTICULARS OF CONTRIBUTORS:}

1. Lecturer, Department of Prosthodontics, MGV's KBH Dental College and Hospital, Nashik.

2. Senior Resident III, Department of Plastic Surgery, Grant Medical College, Sir J.J. Group of Hospitals, Mumbai.

3. Senior Lecturer, Department of Conservative \& Endodontics, SMBT Dental College, Sangamner Taluka, Maharashtra State.
4. Post Graduate Student, Department of Prostshodontics, Rural Dental College, Loni.

5. Reader, Department of Oral \& Maxillofacial Surgery, SMBT Dental College, Sangamner Taluka, Maharashtra.

\section{NAME ADDRESS EMAIL ID OF THE CORRESPONDING AUTHOR:}

Dr. Priyadarshani G. Pawar, Lecturer, MGV's KBH Dental College and Hospital, Nashik.

Email -pri_s77@yahoo.com

Date of Submission: 15/10/2013. Date of Peer Review: 17/10/2013. Date of Acceptance: 31/10/2013. Date of Publishing: 12/11/2013 